\title{
Effect of Early Endoscopic Evaluation With Balloon Dilatation on Preventing Esophagojejunal Strictures Following Total Gastrectomy for Gastric Cancer
}

\author{
Kyeong Woon Choi ${ }^{\mathrm{a}}$, Woo Yong Lee ${ }^{\mathrm{a}, \mathrm{b}}$
}

\begin{abstract}
Background: The present study aims to evaluate the efficacy of early endoscopic evaluation with balloon dilatation performed during nonsymptomatic post-operative period with the intent of preventing benign post-operative esophagojejunal anastomotic strictures in patients who receive total gastrectomy for gastric cancer.
\end{abstract}

Methods: First, we retrospectively compared the prevalence of benign esophagojejunal anastomotic stricture occurred 1 month after total gastrectomy for gastric cancer in early endoscopic evaluated group using balloon dilatation $(\mathrm{n}=61)$ with non-evaluated group $(\mathrm{n}=148)$; and then we reviewed the relationship between benign anastomotic stricture and other clinicopathological parameters, including gender, age, body mass index (BMI), and tumor, node, metastasis (TNM) stage.

Results: The total number of patients was 209, with 139 men and 70 women, and with an average age of 57.8 years. The endoscopic evaluation with or without balloon dilatation on the 10th post-operative day (POD) was performed in 61 out of 209 patients. Of them, benign post-operative anastomotic stricture was found in 17 patients $(27.9 \%)$. In the remaining 148 patients, benign post-operative anastomotic stricture occurred in $14(9.5 \%)$ patients. In the 61 patients who received early endoscopic evaluation, 16 received preventive balloon dilatation at the same time and four of them showed benign stricture $(4 / 16,25 \%)$. Of the remaining 45 patients who was endoscopically evaluated without balloon dilatation, 13 had benign strictures (13/45, $28.9 \%$ ). Preventive endoscopic balloon dilatation (EBD) on the 10th POD was not statistically related to post-operative stricture in a total of 209 patients $(P=0.260)$ and in early gastrofiberscopy (GFS) sub-group $(\mathrm{P}=1.000)$. A multivariable logistic regression analysis showed that pre-operative BMI $(\mathrm{P}=0.014)$ and endoscopy on the 10th POD $(\mathrm{P}=0.001)$ were independent predictors of post-operative anastomotic stricture.

Manuscript submitted October 25, 2018, accepted November 26, 2018

aDepartment of Surgery, Seoul Paik Hospital, Inje University College of Medicine, Seoul, Korea

${ }^{\mathrm{b} C}$ Corresponding Author: Woo Yong Lee, Departments of Surgery, Seoul Paik Hospital, Inje University College of Medicine, 9, Mareunnae-ro, Jung-gu, Seoul 04551, Korea. Email: yongaaa5972@naver.com

doi: https://doi.org/10.14740/jcs370
Conclusions: Endoscopic procedure with balloon dilatation, which we had expected to prevent stenosis by widening the internal diameter of the anastomosis site in early post-operative period after total gastrectomy, does not prevent stenosis but rather promotes post-operative stricture. In addition, the lower the pre-operative BMI is, the more frequent post-operative benign anastomotic stricture will be.

Keywords: Benign stricture; Endoscopic balloon dilatation; Total gastrectomy

\section{Introduction}

Gastric cancer is the second most common cancer [1] and the third leading cause of cancer deaths in Korea, following lung cancer and liver cancer [2]. While the incidence of cancer in the lower third part of the stomach is mildly decreasing, that in the upper third part of the stomach has recently been increasing. As a result, complications, such as anastomotic site bleeding, leakage and benign esophagojejunal stricture, which are possible problems of total gastrectomy, have become issues requiring closer attention than before. Especially benign esophagojejunal strictures may progress immediately or long after surgery, resulting in poor nutritional status and quality of life. Recent studies examining the efficacy of endoscopic balloon dilatation (EBD) for preventing esophageal strictures after endoscopic mucosal resection (EMR) or endoscopic submucosal dissection (ESD) for esophageal cancer have reported positive findings $[3,4]$. Spurred by these results, we decided to examine the potential effect of early endoscopic evaluation with balloon dilatation on preventing anastomotic strictures following total gastrectomy for gastric cancer.

\section{Patients and Methods}

Of 1,865 patients who received gastrectomy for gastric cancer at the Seoul Paik Hospital of Inje University between January 1, 2002 and December 31, 2011, 209 patients who received total gastrectomy were selected for the analysis. In this study, we included the patients who underwent total gastrectomy and did not undergo pre-operative treatment including neoadjuvant therapy. And we excluded patients who had distant metastasis, 
metastatic malignant stricture in anastomosis and other malignant diseases, or those who received previous chemotherapy. All operations were open total gastrectomy with retrocolic Roux-en-Y bypass using a circular stapler (EEA $\left.{ }^{\mathrm{TM}}\right)$.

In this study, stricture was defined as a narrowing of the anastomosis site to such an extent that a 9-mm diameter endoscope could not pass in follow-up endoscopy performed 1 month after surgery. Early gastrofiberscopy (GFS) was defined as an endoscopic examination performed at the 10th post-operative day (POD). Preventive EBD was defined as an EBD performed regardless of the presence of stricture in early GFS (immediately before the anastomosis site heals completely). The preventive EBD procedure was performed using a $15-\mathrm{mm}$ pyloric balloon set, followed by three consecutive ballooning, with increasing pressures from $20 \mathrm{lb} / \mathrm{in}^{2}$ (psi) to $30 \mathrm{~s}, 30 \mathrm{psi}$ to $60 \mathrm{~s}$, and $40 \mathrm{psi}$ to $60 \mathrm{~s}$. The all procedures were performed only in the patients providing consent.

First, all the patients were divided into two groups with or without early GFS. And then the early GFS group was subdivided into two groups with or without preventive EBD. In this way, we performed a subgroup analysis of the early GFS group to investigate the statistical significance of preventive EBD. All the patients underwent follow-up endoscopic examination 1 month after surgery, and then were evaluated for postoperative esophagojejunal anastomotic stricture. The patients' medical records were reviewed retrospectively to examine the relationship between early GFS, preventive EBD and anastomotic stricture. Other clinicopathologic factors, such as sex, age, pre-operative BMI, triglyceride (TG) level, EEA ${ }^{\mathrm{TM}}$ diameter, stage of gastric cancer (early gastric cancer (EGC) vs. advanced gastric cancer (AGC)), TNM stage, and tumor size, were also reviewed. This retrospective study was approved by the institutional review boards of the Seoul Paik Hospital, Inje University College of Medicine.

The statistical analysis was performed using SPSS Statistics software (version 18.0, SPSS Inc., Chicago, IL). Categorical variables were analyzed by using the Chi-square test and Fisher's exact test. In the multivariable analysis, a logistic regression analysis was applied to identify independent clinicopathological factors that were associated with stricture. The results of the statistical tests were considered to be significant when $\mathrm{P}<0.05$.

\section{Results}

The 209 patients were consisted of 139 men and 70 women whose mean age was 57.8 years (22 - 82 years). The mean follow-up period was 3.23 years ( 1 - 10 years). As for the BMI distribution, 91 patients were in the normal range (BMI $\geq 18.5$ and $<23 \mathrm{~kg} / \mathrm{m}^{2}$ ), 10 in the underweight range, and 108 in the overweight or obese range. Two different gauges of EEA ${ }^{\mathrm{TM}}$ were used in the total gastrectomy. A total of 191 patients were treated with a $25-\mathrm{mm}$ gauge and 18 with a $28-\mathrm{mm}$ gauge. All the patients were evaluated for the pre-operative TG level, 173 of whom had a TG level $<150 \mathrm{mg} / \mathrm{dL}$, and 36 had a TG level $\geq 150 \mathrm{mg} / \mathrm{dL}$. As for the stage of gastric cancer, 54 patients had EGC, while 155 patients had AGC. Tumor size was de- termined on the basis of the longest axis of the tumor whose mean size was $6.1 \mathrm{~cm}(0.6-20 \mathrm{~cm})$. A total of 119 patients had a tumor size of $<6 \mathrm{~cm}$ and 90 had a tumor size of $\geq 6 \mathrm{~cm}$. Table 1 shows clinicopathological characteristics of the 209 patients who underwent total gastrectomy. Anastomotic strictures occurred in 31 out of 209 patients (14.9\%). Of them, 14 did not undergo any early endoscopic evaluation, 17 received early GFS, and four of them also received preventive EBD. On the 10th POD, 61 patients agreed to receive early GFS and 16 out of them also agreed to receive preventive EBD. A univariable analysis showed that anastomotic stricture was correlated with BMI $(P=0.031)$ and early GFS $(P=0.001)$. And other clinicopathologic factors such as sex, age, pre-operative BMI, TG level, EEA ${ }^{\mathrm{TM}}$ diameter, stage of gastric cancer, TNM stage and tumor size were not statistically significant (Table 1). In subgroup analysis of the early GFS group, no statistical relationship was found between preventive EBD and anastomotic stricture (odds ratio (OR), 0.821, $\mathrm{P}=1.000$ ) (Table 2). A multivariable logistic regression analysis showed that BMI $(\mathrm{P}=$ $0.014)$ and early GFS $(\mathrm{P}=0.001)$ were independent predictors for anastomotic stricture. The incidence of stricture tended to decrease with increasing pre-operative BMI $(\mathrm{P}=0.014)$ and increase in the early GFS group $(\operatorname{Exp}(B)=3.958, P=0.001)$ (Table 3).

\section{Discussion}

Post-operative benign anastomosis site strictures are usually secondary to edema, early scar formation or chronic inflammation. The occurrence rate of benign stricture at the esophagojejunal anastomotic site ranges from 1.2 to $16 \%$ [5]. Many factors are thought to contribute to the stricture, including additional serosal sutures, anastomosis site leakage, and mismatching of the staple size which sometimes results in esophageal laceration, post-operative delay of oral feeding, granulation at the anastomotic site and circulatory disturbance at the anastomotic site [6]. Polglase et al [7] and Penninckx et al [8] reported that the mechanism underlying stricture may be incomplete anastomosis of the mucous membranes or secondary healing triggered by delayed epithelialization of surgical wounds in the luminal wall involving necrotic and granulation tissues or nonabsorbent staples placed in a circular pattern inhibiting the lumen from sufficiently dilating. Chung [9] investigated blood flow at the anastomosis sites and found that a staple height of $2.3 \mathrm{~mm}(75 \%$ of intestinal thickness) decreased stricture formation and that the ischemia intensified as the staple height decreased. Ezoe et al [4] reported that preventive EBD was effective in preventing esophageal strictures in patients whose esophageal lumen has become defective because of ESD or EMR for esophageal cancer. In their study, preventive EBD was commenced within 1 week after the EMR/ESD and repeated weekly until the complete healing of mucosal defect was observed. Based on these research findings, we have assumed that strictures occur when the lumen of the anastomosis site fails to achieve sufficient diameter during the natural wound healing process. Thus, we thought that by providing early preventive EBD (on the 10th POD, immediately before 
Table 1. Clinicopathological Characteristics of the 209 Patients Who Underwent Total Gastrectomy

\begin{tabular}{|c|c|c|c|c|}
\hline \multirow{2}{*}{ Variable } & \multicolumn{2}{|c|}{ Delayed stricture, $\mathrm{n}=\mathbf{2 0 9}$} & \multirow{2}{*}{ P-value } & \multirow{2}{*}{ Number $(\%), \mathbf{n}=209$} \\
\hline & No $(\%) n=178$ & Yes $(\%) n=31$ & & \\
\hline \multicolumn{5}{|l|}{ Age } \\
\hline$\geq 60$ & $88(49.4)$ & $15(48.4)$ & & $103(49.3)$ \\
\hline \multicolumn{5}{|l|}{ Sex } \\
\hline Male & $122(68.5)$ & $17(54.8)$ & 0.136 & $139(66.5)$ \\
\hline \multicolumn{5}{|c|}{ Body mass index $\left(\mathrm{kg} / \mathrm{m}^{2}\right)$} \\
\hline$<18.5$ & $5(2.8)$ & $5(16.2)$ & 0.031 & $10(4.8)$ \\
\hline$\geq 18.5$ and $<23$ & $78(43.8)$ & $13(41.9)$ & & $91(43.5)$ \\
\hline$\geq 23$ & $95(53.4)$ & $13(41.9)$ & & $108(51.7)$ \\
\hline \multicolumn{5}{|l|}{$\mathrm{EEA}^{\mathrm{TM}}$ size $(\mathrm{mm})$} \\
\hline$<150$ & $146(82.0)$ & $27(87.1))$ & 0.267 & $173(82.8)$ \\
\hline$\geq 150$ & $32(18.0)$ & $4(2.9)$ & & $36(17.2)$ \\
\hline \multicolumn{5}{|l|}{ Stage } \\
\hline Early & $46(25.8)$ & $8(25.8)$ & 0.997 & $54(25.8)$ \\
\hline Advanced & $132(74.2)$ & $23(74.2)$ & & $155(74.2)$ \\
\hline \multicolumn{5}{|l|}{ T stage } \\
\hline $\mathrm{T} 1$ & $43(24.2)$ & $8(25.8)$ & 0.815 & $51(24.4)$ \\
\hline $\mathrm{T} 2$ & $21(11.8)$ & $2(6.5)$ & & $23(11.0)$ \\
\hline $\mathrm{T} 3$ & $51(28.7)$ & $9(29.0)$ & & $60(28.7)$ \\
\hline $\mathrm{T} 4$ & $63(35.3)$ & $12(38.7)$ & & 75 (35.9) \\
\hline M0 & $165(92.7)$ & $28(90.3)$ & 0.712 & $193(92.3)$ \\
\hline M1 & $13(7.3)$ & $3(9.7)$ & & $16(7.7)$ \\
\hline \multicolumn{5}{|l|}{ Tumor size } \\
\hline$<6 \mathrm{~cm}$ & $98(55.1)$ & $21(67.7)$ & 0.188 & $119(56.9)$ \\
\hline$\geq 6 \mathrm{~cm}$ & $80(44.9)$ & $10(32.3)$ & & $90(43.1)$ \\
\hline \multicolumn{5}{|l|}{ Early $^{\mathrm{a}} \mathrm{GFS}^{\mathrm{b}}$} \\
\hline No & $134(75.3)$ & $14(45.2)$ & 0.001 & $148(70.8)$ \\
\hline Yes & $44(24.7)$ & $17(54.8)$ & & $61(29.2)$ \\
\hline \multicolumn{5}{|c|}{ Earlya balloon dilatation } \\
\hline No & $166(93.3)$ & $27(87.1)$ & 0.266 & $193(92.3)$ \\
\hline Yes & $12(6.7)$ & $4(2.9)$ & & $16(7.7)$ \\
\hline \multicolumn{5}{|l|}{ Delayed $^{\mathrm{c}}$ stricture } \\
\hline No & & & & $178(85.1)$ \\
\hline Yes & & & & $31(14.9)$ \\
\hline
\end{tabular}

Tumor stages are based on the TNM classification system from the seventh edition of the Union for International Cancer Control/American Joint Committee on Cancer. a"Early" means the 10th POD; 'bFS: gastrofiberscopy; "“Delayed" means 1 month after surgery. 
Table 2. Association Between EBD and Delayed Stricture in Subgroup Analysis of Early GFS Group ${ }^{a}$

\begin{tabular}{|c|c|c|c|c|}
\hline \multirow{2}{*}{ Early balloon dilatation (in Early GFS group, $n=61$ ) } & \multicolumn{2}{|c|}{ Stricture } & \multirow{2}{*}{ OR $(95 \% \mathrm{CI})$} & \multirow{2}{*}{ P-value } \\
\hline & Positive $(n=17)$ & Negative $(n=44)$ & & \\
\hline Yes $(n=16)$ & 4 & 12 & $0.821(0.223-3.018)$ & 1 \\
\hline
\end{tabular}

aFisher's exact test.

the healing process is complete), we can reduce the incidence of stricture as reported by Ezoe's study [4] by inhibiting early scar formation and securing sufficient anastomotic diameter. However, in our study, preventive EBD was not a statistically significant factor and early GFS itself was statistically associated with the increased risk for stricture.

The first reason for the difference between the results of our study and those of Ezoe's study [4] is the anatomical differences between the two models. Ezoe's model is an esophagus that was not completely dissected. The esophagus in this model had only internal defects, but the original external integrity was not impaired. The model of our study, on the other hand, was the anastomosis of esophagus and jejunum with histologically different structures, which are connected after complete dissection. The healing process in this anastomosis between esophagus and jejunum may be different from that of a single organ. The healing time in anastomosis is considered to be longer than the partial injury of a single organ. Direct contact of the scope with the anastomotic region and endoscopic air burden during the early GFS performed prior to complete wound healing would likely induce secondary damage and delay healing at the anastomosis site. These factors are thought to induce stricture. Considering these points, preventive EBD may be effective in preventing stricture in partially and internally damaged tissue [4], but if it is performed before complete healing in the anastomosis between complete and external dissected tissues, it seems to promote stricture.

The second reason to consider is the ballooning schedule. In Ezoe's model [4], ballooning was repeated weekly from 7 days after EMR or ESD until complete healing was confirmed. In our study, GFS and ballooning were performed only once, 10 days after surgery. Given the cost and efficiency issues, repetitive GFS and ballooning were difficult to implement. If these problems could be solved and repetitive GFS and ballooning were performed after surgery, the results may have been different. However, based only on the result of this study, at least GFS and ballooning on the 10th POD are not effective in preventing post-operative stenosis, but rather promote postoperative stricture.

To date, there has been little understanding of the normal course of intestinal healing, and little is known about pathobiology as to how it leads to stricture formation. It is thought that a more holistic understanding of stricture formation will require detailed and dynamic studies of anastomosis site healing through direct examination with tissue sampling and analysis.

\section{Conclusions}

The development of esophagojejunal strictures after total gastrectomy for gastric cancer is statistically associated with preoperative BMI and early GFS with/without balloon dilatation. The preventive EBD following total gastrectomy does not significantly prevent benign esophagojejunal strictures.

\section{Funding}

None.

\section{Conflict of Interest}

All authors declare that there is no conflict of interest.

\section{Abbreviations}

BMI: body mass index; TNM: tumor, node, metastasis; POD: post-operative day; EBD: endoscopic balloon dilatation; GFS: gastrofiberscopy; EMR: endoscopic mucosal resection; ESD: endoscopic submucosal dissection; TG: triglyceride; EGC: early gastric cancer; AGC: advanced gastric cancer; OR: odds

Table 3. Multivariable Analysis Showing Factors Associated With Delayed Stricture in 209 Patients $^{a}$

\begin{tabular}{|c|c|c|c|c|c|c|c|}
\hline Variable & & Total $(\%), n=209$ & No stricture (\%) & Stricture & $\operatorname{Exp}(B)$ & $95 \%$ CI & P-value \\
\hline \multirow[t]{2}{*}{ BMI } & $<18.5$ & $10(4.8)$ & $5(2.8)$ & $5(16.2)$ & 1 & & 0.014 \\
\hline & $\geq 18.5$ and $<23$ & $91(43.5)$ & $78(43.8)$ & $13(41.9)$ & 0.132 & $0.031-0.567$ & \\
\hline \multirow[t]{2}{*}{ Early GFS } & No & $148(70.8)$ & $134(75.2)$ & $14(45.2)$ & 1 & & 0.001 \\
\hline & Yes & $61(29.2)$ & $44(24.8)$ & $17(54.8)$ & 3.958 & $1.743-8.991$ & \\
\hline
\end{tabular}

aLogistic regression analysis. 
ratio

\section{References}

1. Ministry of Health and Welfare, Cancer registration data. (Approved Statistics No. 11744-2011).

2. Statistics Korea, Causes of death data. Approved Statistics No. 10154-2013).

3. Wang $\mathrm{C}, \mathrm{Lu} \mathrm{X}, \mathrm{Chen} \mathrm{P}$. Clinical value of preventive balloon dilatation for esophageal stricture. Exp Ther Med. 2013;5(1):292-294.

4. Ezoe Y, Muto M, Horimatsu T, Morita S, Miyamoto S, Mochizuki S, Minashi K, et al. Efficacy of preventive endoscopic balloon dilation for esophageal stricture after endoscopic resection. J Clin Gastroenterol. 2011;45(3):222227.
5. Peifer KJ, Shiels AJ, Azar R, Rivera RE, Eagon JC, Jonnalagadda S. Successful endoscopic management of gastrojejunal anastomotic strictures after Roux-en-Y gastric bypass. Gastrointest Endosc. 2007;66(2):248-252.

6. Inagake M, Yamane T, Kitao Y, Okuzumi J, Kuwata K, Yamaguchi T, Oya K, et al. Balloon dilatation for anastomotic stricture after upper gastro-intestinal surgery. World J Surg. 1992;16(3):541-544.

7. Polglase AL, Hughes ES, McDermott FT, Pihl E, Burke FR. A comparison of end-to-end staple and suture colorectal anastomosis in the dog. Surg Gynecol Obstet. 1981;152(6):792-796.

8. Penninckx FM, Kerremans RP, Geboes KJ. The healing of single- and double-row stapled circular anastomosis. Dis Colon Rectum. 1984;27:714-719.

9. Chung RS. Blood flow in colonic anastomoses. Effect of stapling and suturing. Ann Surg. 1987;206(3):335-339. 